\title{
LHC BEAM INSTRUMENTATION
}

\author{
J.Bosser, C. Bovet, C. Fischer, R. Jung, H. Koziol, H. Schmickler, L. Vos \\ CERN, Geneva, Switzerland
}

\section{Abstract}

Six years before the scheduled commissioning of the LHC at CERN, the basic list of beam instruments has been established. This early date is needed due to the impact of the mechanical design of some detectors (mainly the beam position detectors) on the cryogenic part of the machine as well as for other projects due to the long $R \& D$ period (emittance measurements, tune and chromaticity diagnostics and control). This paper gives a detailed overview of the basic requirements and specifications of all beam instruments foreseen for transfer lines and main rings [1].

\section{INTRODUCTION}

Underneath the super-conducting dipoles around the LHC ring the annual radiation dose will be as low as $1 \mathrm{~Gy}$. This is due to the extremely effective halo cleaning system necessary to prevent magnet quenches. For beam instrumentation this means that front-end electronics can be spread around the circumference, avoiding long and expensive cables.

Table 1. Summary list of LHC instruments [2]

\begin{tabular}{|c|c|c|}
\hline \multicolumn{2}{|c|}{ Transfer lines between SPS and LHC (5 km) } & Nb \\
\hline \multicolumn{2}{|c|}{ Beam position monitors (using LEP buttons) } & 110 \\
\hline \multicolumn{2}{|l|}{ Beam current transformers } & 4 \\
\hline \multicolumn{2}{|l|}{ Beam loss monitors } & 20 \\
\hline \multicolumn{2}{|c|}{ Screens using optical transition radiation } & 20 \\
\hline \multicolumn{3}{|l|}{ LHC main ring } \\
\hline \multicolumn{2}{|c|}{ Injection screens with optical transition radiation } & 16 \\
\hline Betatron matching, thin OTR foils & $11 \mathrm{kHz}$ & 2 \\
\hline Beam position monitors & $40 \mathrm{MHz}$ & 988 \\
\hline Beam loss monitors distributed & $150 \mathrm{kHz}$ & 4000 \\
\hline BLM for halo cleaning (ACEM) & $40 \mathrm{MHz}$ & 10 \\
\hline \multicolumn{2}{|l|}{ Beam current transformer systems } & 2 \\
\hline \multicolumn{2}{|l|}{ Wire scanners for beam size calibration } & 4 \\
\hline Synchrotron light telescopes & $\bullet 40 \mathrm{MHz}$ & 4 \\
\hline \multicolumn{2}{|c|}{ Beam size detectors (R\&D on the way) } & 4 \\
\hline \multicolumn{2}{|c|}{ Tune measurement systems using 4 kickers } & 2 \\
\hline \multicolumn{2}{|c|}{ Chromaticity (from head-tail phase shift) } & 2 \\
\hline \multicolumn{2}{|l|}{ Schottky pick-ups } & 4 \\
\hline \multicolumn{2}{|c|}{ On-line control systems of Q,Q' and closed orbit } & 6 \\
\hline \multicolumn{3}{|l|}{ Dump lines } \\
\hline \multicolumn{2}{|l|}{ Screens } & 10 \\
\hline \multicolumn{2}{|l|}{ Beam current transformers } & 2 \\
\hline
\end{tabular}

For distributed monitors like beam position (BPM) and beam loss (BLM) data collection will be done through some 240 acquisition crates located at $30 \mathrm{~m}$ from the monitors.

\section{BEAM POSITION MEASUREMENT}

Trajectory and closed orbit measurements are vital for commissioning and operation of accelerators. To achieve high luminosity, the azimuthal beam distribution becomes very complex, so that various phenomena (beam-beam forces, wake fields) strongly affect the orbits of individual bunches. Hence a system with high bandwidth capable of measuring the transverse position of each bunch is desirable. In LHC it is foreseen to install BPM's reading both planes at each lattice quadrupole which makes a total of 988 pick-ups spread over the two rings of $27 \mathrm{~km}$ circumference.

\subsection{Mechanical design}

Pick-ups will be made of four buttons yielding enough signal amplitude to measure the position of a pilot bunch of $5 \times 10$ protons. Most BPMs will be located near supraconducting quadrupoles with local electronics mounted outside the cryostat and receiving beam signals through coaxial cables of $1.3 \mathrm{~m}$ length.

\subsection{Signal treatment}

In the context of the development of an orbit system for the LHC a high bandwidth was achieved by extending the principle of the narrow-band normaliser with phase detection to a wide-band time normaliser[3],[4]. The principle of this new circuit is shown in Fig. 1.

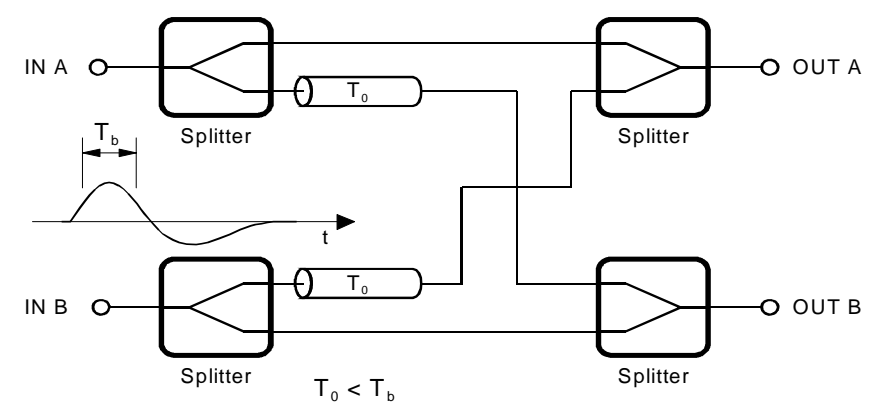

Figure 1. Principle of the wide-band normaliser

Results obtained in the laboratory on the first prototype prove that a linearity of $0.2 \%$ can be achieved over the full aperture, see Fig. 2. A bandwidth of more than $40 \mathrm{MHz}$ and a dynamic range of $50 \mathrm{~dB}$ was achieved. 


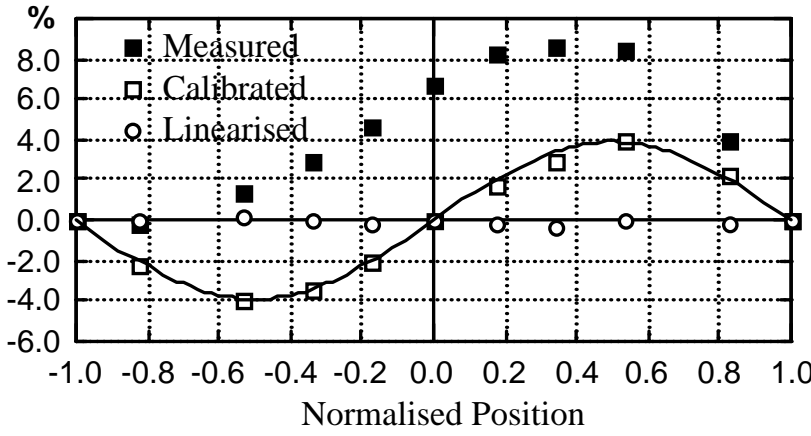

Figure 2. Position linearity with the wide-band normaliser

\subsection{BPM performances}

Absolute accuracy shown in Table 2 is mainly determined by alignment precision between the magnetic centre of the quadrupole and the electrical centre of the BPM. For the pilot bunch this is determining, while for nominal bunches the offset will be eliminated by using the K-modulation technique[5].

Table 2. BPM performances

\begin{tabular}{|l|c|c|c|c|}
\hline \multicolumn{1}{|c|}{ Bunch type } & \multicolumn{2}{c|}{ Pilot } & \multicolumn{2}{c|}{ Nominal } \\
\hline Mode & Traject. & Orbit & Traject. & Orbit \\
\hline Absolute accuracy & $1.5 \mathrm{~mm}$ & $1 \mathrm{~mm}$ & $150 \mu \mathrm{m}$ & $100 \mu \mathrm{m}$ \\
\hline Resolution & $0.5 \mathrm{~mm}$ & $0.2 \mathrm{~mm}$ & $50 \mu \mathrm{m}$ & $5 \mu \mathrm{m}$ \\
\hline
\end{tabular}

\section{TRANSVERSE BEAM PROFILES}

Keeping the highest possible transverse density through the whole injector chain up to the top energy in the LHC is one of the major goals to optimise luminosity. For that reason OTR screens (producing optical transition radiation) have been tested and will be provided in transfer lines. They are made of Ti or $\mathrm{Al}$ foils, a few $\mu \mathrm{m}$ thick and are viewed with high-resolution CCD cameras.

\subsection{Injection matching}

A new method has been introduced for betatron matching into a circular machine [6] which consists in using a thin detector to measure the beam size for several revolutions before dumping the beam. The detectors can be either secondary emission grids or OTR screens. When r.m.s beam size is beating by less than $\pm 10 \%$, which is easy to check, this means that the emittance blow-up by filamentation will be less than $1 \%$.

\subsection{Wire scanners}

Wire scanners currently used in LEP will be very useful to measure beam profiles with great accuracy since the wire position can be measured to $\pm 4 \mu \mathrm{m}$. Still they cannot be used with more than 200 nominal bunches for the wire to survive [7] and therefore cannot be used operationally, but will serve to calibrate the other types of monitors described in the next two paragraphs.

\section{$3.3 R \& D$ on beam profile monitors}

Three types of monitors using the residual gas in the machine vacuum are presently undergoing tests in the SPS with proton bunches separated by $25 \mathrm{~ns}$ :

- An ionisation profile monitor constructed at DESY will be tested with an additional magnetic field which should serve to collect electrons with minimum space charge blurring;

- Another monitor collects ions accelerated by the beam's electrostatic potential without any other external field. The velocity distribution of those ions escaping the vacuum chamber through a slit, is measured in a magnetic spectrometer and the beam size can be deduced from the velocity distribution;

- The third monitor observes the light emitted by residual gas molecules excited by the beam. Selecting a gas with fast decay allows to get a clean transverse beam profile and a local bump of nitrogen pressure of less than $10^{-6}$ torr is sufficient to collect enough photons in $20 \mathrm{~ms}$.

A fourth monitor is also giving promising results: it consists of an ion-gun accelerating a beam of $\mathrm{Xe}^{+}$or $\mathrm{Kr}^{+}$to an energy of $2 \mathrm{keV}$ which is sent at a right angle to the proton beam. The deflection angle of the ion beam is measured as a function of the ion beam position and the derivative of this function gives the proton beam profile.

At top energy the r.m.s. beam size will be of some $400 \mu \mathrm{m}$ and in order to perceive an emittance blow-up of a few percent the beam size should be measured to $1-2 \%$ relative accuracy. The best of the four preceding monitors will be retained for operational use.

\subsection{Synchrotron radiation telescopes}

The use of synchrotron radiation to measure transverse beam profiles has been analysed [8] and seems delicate at injection energy where a special short magnet is needed to produce enough power in the near infra-red region. This power will rise by 5 orders of magnitude during ramping, which is not easy to master at the detector level. This great dynamic range stems from the fact that useful photons belong to the higher part of the spectrum, well beyond the critical energy. Another possibility at injection energy would be to use the enhanced synchtrotron radiation power produced in the fringe field of the same dipole. At top energy, in order to determine the beam sizes during physics, a UV telescope receiving light from a $3.5 \mathrm{~T}$ dipole could monitor permanently with a CCD camera the transverse beam density. Using a wavelength of $200 \mathrm{~nm}$, the diffraction spot sizes will be of 300/130 $\mu \mathrm{m}$ for $\mathrm{H} / \mathrm{V}$ profiles respectively, to be deconvoluted from beam sizes of $890 / 480 \mu \mathrm{m}$.

For the detection of individual bunch emittances a photomultiplier with 16 anodes can acquire bunch sizes at a frequency of $40 \mathrm{MHz}$. 


\section{TUNE AND CHROMATICTY MEASUREMENTS}

Tune, chromaticity and transverse coupling need to be controlled throughout critical phases of the machine cycle, in particular during ramping and beta-squeezing.

\subsection{Use of the transverse feedback}

Signals may be injected into the feedback loop, either white noise for broad-band spectral analysis, or a sweptfrequency sine-wave. The latter allows the measurement of the beam transfer-function, i.e. the response in amplitude and phase.

\subsection{Coherent oscillations created with a kicker}

One would perhaps not include this method, needing special kickers, were it not for other possibilities that it provides:

- coupling is easily measured, using a single kick in one plane, and resonances can be studied, by "tickling" the beam.

- very importantly, kicking to oscillation amplitudes greater than the size of the beam, which is possible at injection energy, and using two pick-ups an odd multiple of quarter-betatron periods apart, allows to observe long-term evolution in transverse phase space, for study of the dynamic aperture. Therefore additional single-turn kickers with enough strength to displace the beams at $7 \mathrm{TeV}$ by $8 \sigma$ will be installed.

- finally, the kicks can serve for the measurement of chromaticity, via the head-tail phase shift (next paragraph).

\subsection{Head-tail chromaticity measurements}

In addition to the classical methods of measuring chromaticty [9] a new method has been developed at CERN that allows the chromaticity to be calculated after several hundred turns from the turn-by-turn position data of a single bunch after transverse excitation.

This so-called head-tail chromaticity measurement [10] relies on the fact that for non-zero chromaticity a dephasing/re-phasing occurs between the head and tail betatron motion of a bunch with the synchrotron frequency. Immediately after the exciting kick, head and tail oscillate in phase. On subsequent turns, the head and tail de-phase due to chromaticity, reaching a maximum phase difference after half a synchrotron period. By measuring the turn-by-turn position data for two longitudinal positions in a bunch it is possible to extract the phase difference between the head and the tail oscillations and so to determine the chromaticity.

This technique has several advantages over other methods. Firstly, only a few hundred turns of data is required, which allows for short acquisition times. Secondly, unlike many other methods, no knowledge of the betatron tune is required, and there is also very little dependence on energy for machines operating well above transition.

\section{INTENSITY MEASUREMENTS}

Beam transformers and many other beam diagnostics will be located where the beam separation is $0.4 \mathrm{~m}$.

\subsection{Beam current transformers}

In order to obtain a bandwidth of the order of $1 \mathrm{GHz}$, current transformers of a single-turn variant or wall-currentmonitor will be needed. Ferrite loading will extend the lower cut-off frequency.

Once injection is completed and the beams are circulating, the fast beam transformers will serve to determine the number of protons contained in each of the 2835 bunches, with $1 \%$ precision. For practical reasons, this will not be done on a single turn, which is acceptable as this information does not require continuous surveillance.

\subsection{DC beam current transformers}

These monitors are based on the principle of the magnetic amplifier, using a null-method, and will measure the intensity, or current, of the circulating beams.

DC beam transformers currently reach a resolution of 1 $\mu \mathrm{A}$, corresponding to $5 \times 10^{8}$ protons in an LHC ring. The output drift is about $1 \mu \mathrm{A}$ over $10 \mathrm{~s}, 2 \mu \mathrm{A}$ over 1 day, and $3 \mu \mathrm{A}$ over a week, but a significant improvement may be achieved before the LHC start-up. $A$ resolution of $1 \mu \mathrm{A}$ on the one hand, and a nominal beam intensity of $0.54 \mathrm{~A}$ on the other, means a dynamic range of $5 \times 10^{5}$, requiring an analogue to digital conversion of 21 bits.

\section{ACKNOWLEDGEMENTS}

The authors would like to thank warmly all members of PS-BD and of SL-BI who have contributed to this project.

\section{REFERENCES}

[1] J. Bosser et al., "LHC Design Report, chapter on Beam Instrumentation",LHC Project Report to be issued in 1999.

[2] C. Fischer, "A review of the LHC beam instrumentation", LHC Project Note 144, Apr. 1998.

[3] D. Cocq, "The wide-band normaliser - a new circuit to measure bunch position ...", NIM A 416 (1998) pp. 1-8.

[4] D. Cocq, H. Schmickler and G. Vismara, "A new wideband time normaliser circuit ... ", HEACC, 1998.

[5] B. Dehning et al., "Dynamic beam based calibration of beam position monitors", EPAC'98, pp. 430-433.

[6] C. Bovet and R. Jung, "A new diagnostic for betatron phase space matching", LHC Project Report 3, Rev., 1996.

[7] J. Bosser and C. Bovet, "Wire scanners for the LHC" LHC Project Note 108, Rev., Oct. 1997.

[8] J. Bosser et al., "Preliminary studies on a profile monitor for the LHC using s.r.", LHC/Note 192, June 1992.

[9] H.Schmickler, "Diagnostics and control of the time evolution of beam parameters", DIPAC 1997, pp. 43-48.

[10] D.Cocq, R.Jones, H.Schmickler, "The measurement of chromaticity via head-tail phase shift”, BIW 1998, pp. 281. 